\title{
Spatial-temporal features for smoke detections on video images
}

\author{
Li Ma ${ }^{1}$ \\ (Dept.of information engineering, Shengda Trade Economics \& management college \\ of Zhengzhou 451191, China)
}

\begin{abstract}
Three temporal-space features are proposed in this paper on video images for smoke detections on the issue of dynamic textures, motion patterns and contour variations. Considering that the previous methods for smoke detection provides global descriptions on dynamic features, but its illustrations do not well indicate the temporal correlation among frames, the novel spatial temporal descriptors among neighboring frames are proposed. Our method combines local and global descriptions in the temporal-space domains, where concatenated histograms of LBP (local binary pattern) are utilized for dynamic texture descriptors, the $2 \mathrm{D}$ mutual information for motion disorders and the information entropy for contour variations. The experimental results show that the proposed spatial-temporal features could effectively discriminate smoke and the non-smoke objects.
\end{abstract}

Keywords: spatial-temporal features; concatenated LBP histograms; 2D mutual information; contour variations

\section{Introduction}

The early detections of fire smokes are significant for forest safety monitoring. Fire smokes on video images appear some dynamic features in particular: motion disorders (each pixels with random movements), contour variations (contour changes with time) and dynamic textures (changes of gray-intensity patterns).

In recent years, much of research attention is focused on temporal-space features for smoke detections on video images. Some previous descriptions for smoke temporal features are mainly on global features with time such as the area expansibility and contour irregularity, which haven't given descriptions on temporal

\footnotetext{
1 L. Ma $(\bowtie)$

Shengda Trade Economics \& management college of Zhengzhou 451191, China

e-mail: mali0328@hotmail.com
} 
correlation among frames for contour variations and motion patterns ${ }^{[1]}$. And there is some work for motion disorders by using multi-resolution histograms on velocities and accelerations of smoke pixels but with huge computations ${ }^{[2]}$. As for dynamic texture concerned, there are some parametric (liner dynamic model) and non-parametric models (Bayesian networks) in literatures ${ }^{[3]}$. These models all need huge amount of learning procedures. It is well known that LBP model is a good local descriptor for texture feature on space domain but no temporal correlation among frames. In this paper temporal-space features on local and global combinations for smoke dynamic descriptions are presented with the concatenated histograms of LBP for dynamic textures (extension of original LBP), 2D mutual information for motion disorders and information entropy for contour variations using video images. Experimental results show the effectiveness of the proposed approaches.

The article is organized in the following: dynamic texture description in section 2 , descriptors on motion disorders and contour variations are illustrated in section 3 . Finally they are the experimental results and conclusion.

\section{Temporal-space Model of Dynamic Textures}

Dynamic texture is a key feature for discriminating fire smokes from other objects. LBP model is an effective descriptor for object textures based on local intensity patterns ${ }^{[4]}$. The LBP calculation is given in Eq. (1).

$$
\operatorname{LBP}_{P, R}\left(x_{c}, y_{c}\right)=\sum_{p=0}^{p-1} s\left(g_{p}-g_{c}\right) 2^{p}
$$

Where $\left(x_{c}, y_{c}\right)$ is a current coordinate of a pixel, and $P$ indicates the number of pixels on a circle with radius $R . \mathrm{s}(\mathrm{x})$, the impulse function. The $g_{p}$ and $g_{c}$ indicate the gray intensity of a pixel on the circle or at center $\left(x_{c}, y_{c}\right)$ respectively ${ }^{[5]}$. The procedure of the LBP calculation of any pixel on an image is shown in Fig. 1 where in a $3 \times 3$ neighborhood a pixel would take the value of one if its intensity is larger than $g_{c}$, otherwise zero instead. Then the binary code is transformed to a decimal value called the LBP value of the current point $\left(x_{c}, y_{c}\right)$.

Suppose a video image sequence $G_{i}, i=1,2, \cdots, n$, the LBP descriptions for dynamic textures are considered on three orthogonal planes: XY, XT and YT. For any pixels at current image $G_{i}, i=1,2, \cdots, n$, there are three neighborhoods centered at the pixels on XY, XT and YT shown in Fig.2. Based on the Eq.(1), three LBP values are produced for the three orthogonal planes for each pixels on the image sequences. The 
three LBP sequences of the three different planes are called XY-LBP, XT-LBP and YT-LBP respectively which describes the textural changes in the spatial and temporal domains of video sequences. Fig.2 presents the concatenated histograms of LBPs on the three orthogonal planes by which the texture patterns are extracted in space-temporal domains.

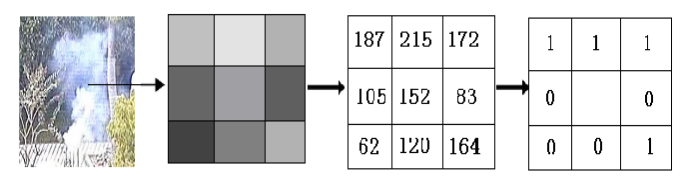

$(11101000)_{10}=232$

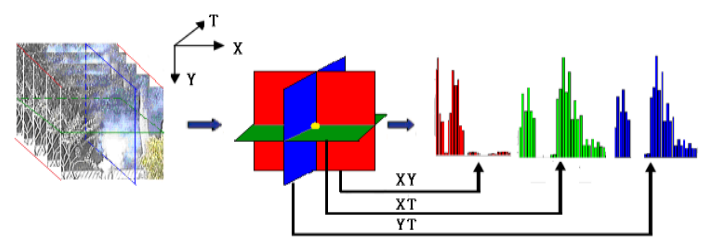

Fig.1 the procedure of LBP coding

Fig.2 concatenated LBP

histograms on three planes

\section{Temporal-space Features for Motion and Contour Variations}

Motion and contour variation are significant temporal-space features for smoke detections. In this paper, two-dimensional mutual information is utilized for measuring the relativity of velocities and orientations of all pixels on candidate smoke regions among neighboring frames in terms of temporal-space domains. Meanwhile, the entropy of the difference in the angle of contingence for any pixels on the contours of the ROI regions of neighboring frames is presented to describe the contour variations.

\subsection{Temporal-space Feature-Two-dimensional Mutual Information}

The two-dimensional mutual information of pixel' velocities and orientations on the candidate smoke regions could measure the motion relativity among sequential images in temporal-space domains. In this paper, the method of L-K optical flow is firstly used for calculating the optical flow field $V=(u, v)^{T}$ for video images. 
where $u$ and $v$ are the horizontal and vertical component of a pixel velocity. The magnitude of velocity $M=\sqrt{u^{2}+v^{2}}$ is quantified as $\mathrm{m}$ in Eq.(2) to reduce computational complexity and the orientation of velocity $f$ is quantified into eight directions.

$$
\mathrm{m}=\left\{\begin{array}{cc}
1, & \mathrm{M}<0.5 \\
\mathrm{k}+1, & 2.5 \mathrm{k}-2 \leq \mathrm{M}<2.5 \mathrm{k}+0.5 \\
8, & \mathrm{M} \geq 15.5
\end{array} \quad k \in\{1,2,3,4,\right.
$$

For any candidate smoke region, a two-dimensional histogram, called a two-dimensional distribution $X$, is formed on magnitudes of velocities $m=\left\{m_{1}, m_{2}, \ldots, m_{S}\right\}$ and orientations $f=\left\{f_{1}, f_{2}, \ldots, f_{N}\right\}$ of all pixels in the region, where $\mathrm{S}$ and $\mathrm{N}$ are the maximum value of $\mathrm{m}$ and $f$ respectively. The mutual information based on magnitudes and orientations of all pixels could indicate the relevancy measure of motion patterns $X_{n}$ and $X_{n+1}$ on two candidate regions either in the nth or the $(n+1)$ th frames. The mutual information of two candidate regions on motion disorders in neighboring frames is presented in Eq.(3).

$$
I\left(X_{n}, X_{n+1}, n\right)=\sum_{i} \sum_{j} \sum_{k} \sum_{l} p\left(\left(m_{i}, f_{j}\right)^{n},\left(m_{k}, f_{l}\right)^{n+1}\right) \times \log \left(\frac{p\left(\left(m_{i}, f_{j}\right)^{n},\left(m_{k}, f_{l}\right)^{n+1}\right)}{p_{n}\left(m_{i}, f_{j}\right) \times p_{n+1}\left(m_{k}, f_{l}\right)}\right)
$$

where $I\left(X_{n}, X_{n+1}, n\right)$ is the two-dimensional mutual information value of the candidate smoke region in the nth frame. $X_{n}$ and $X_{n+1}$, the distribution of motion magnitudes and directions in the nth and $(\mathrm{n}+1)$ th frame. $p_{n}\left(m_{i}, f_{j}\right)$ is the possibility of $\left(m_{i}, f_{j}\right)$ in $X_{n}$ and $p\left(\left(m_{i}, f_{j}\right)^{n},\left(m_{k}, f_{l}\right)^{n+1}\right)$, the joint probability of $X_{n}$ and $X_{n+1}$.

\subsection{Temporal-space Description for Contour Variations}

The entropy measure of the difference in the angle of contingence for any pixels on the contours of the candidate regions of neighboring frames is illustrated in this paper 
for measuring the degree of contour variations with time. The diagram of contour variations is given in Fig.3, where $P$ is the angle between a horizontal direction and the line from a contour point $M$ to center point $O, P=\left\{1^{0}, 2^{0}, \ldots, 360^{\circ}\right\} \cdot Q_{k}$ is the angle of contingent of a contour point $M$ in the kth frame, and $\Delta Q_{k}$ is the difference of $Q$ on contour point $M$ between the $(\mathrm{k}+1)$ th and the kth frame with equal value of $P$. In this way, a entropy of the distribution of $\Delta Q_{k}$ on contour, indicated as $H\left(\Delta Q_{k}\right)$ in Eq.(4), illustrates the degree of a contour variation in neighboring frames. An entropy sequence of $\left\{H\left(\Delta \mathrm{Q}_{\mathrm{k}}\right), k=1,2, \cdots, n\right\}$ shows how the shape of a contour changes in temporal-space domain.

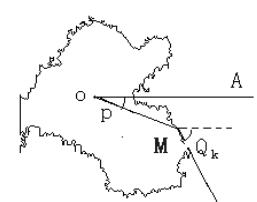

(a) a contour in the kth frame frame

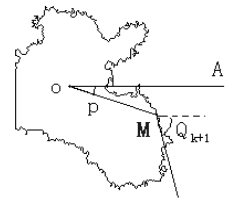

(b)a contour in the $(\mathrm{k}+1)$ th

Fig. 3 a diagram of smoke contour variations

$$
H\left(\Delta Q_{k}\right)=\sum_{\Delta Q_{k}=180}^{180} p_{\Delta Q_{k}} \log _{2} \frac{1}{p_{\Delta Q_{k}}}=-\sum_{i=-180}^{180} p_{\Delta Q_{k}} \log _{2} p_{\Delta Q_{k}}
$$

where $p_{\Delta Q_{k}}$ is the possibility of $\Delta Q_{k}, \quad \Delta Q_{k} \in\left[-180^{\circ}, 180^{\circ}\right]$.

\section{Experimental Results}

Some video images with smoke or non-smoke objects are first taken in open environments and then efficiency of the proposed descriptions is validated in platform Matlab. The Experiments are divided into three parts: the temporal-space LBP model for dynamic textures; two dimensional mutual information for object 
motions; object contour variation in time-space field. Some video frames with cars in white and passengers wearing white shirts are selected in non-smoke set for comparisons.

For dynamic textures based on LBP, some video frames with smokes or non-smokes are firstly segmented and then the histogram distributions of temporal-space LBP are extracted in candidate smoke regions where each video fragments has length of ten. Experimental results for concatenated histograms of XY-LBP, XT-LBP and YT-LBP for different objects mentioned above are shown in Fig.4.
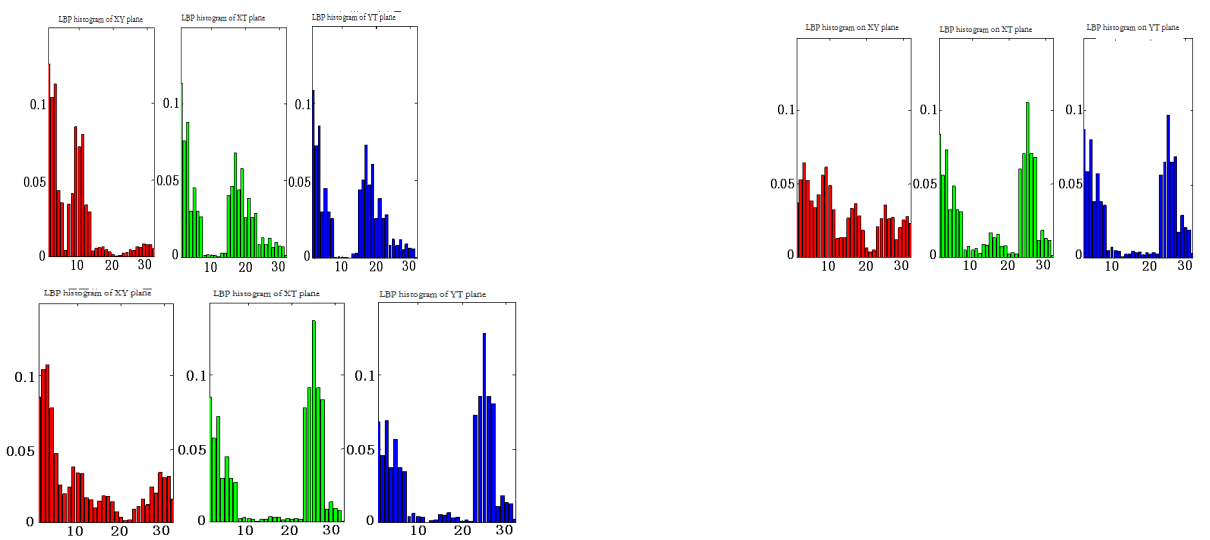

$\begin{array}{lll}\text { (a) LBP histograms for smokes } & \text { (b) LBP histograms for cars } & \text { (c) LBP histograms for passengers }\end{array}$

Fig.4 concatenated LBP histograms in XY, XT and YT planes

It is indicated from Fig.4 that histogram distributions in three orthogonal planes have different patterns among different objects. The LBP distributions for smokes are mainly located in $[0,13]$ of XY plane, $[0,7]$ and $[16,23]$ of XT and YT planes, while the LBP histogram distributions of cars and passengers are quite different with that of smokes as they mainly located in [0,7] and [24,28] of XT and YT planes. It seems that concatenated histograms of XY-LBP, XT-LBP and YT-LBP could be an effective descriptor for dynamic textures to discriminate smokes from others.

The second experiment is about motion features in candidate smoke regions based on the 2D mutual information. To reduce computational burden, a smaller square region in upper left of the candidate smoke region's center is selected for the computations of the Eq.(3). The experimental results are shown in Fig.5. The optical flow field of an original image of Fig.5 (a) is shown in Fig.5 (b) where '*' indicates the center of the candidate smoke region and a square is the small region for 
calculations of the 2D mutual information. Profiles of the two dimensional information for 9 objects are given in Fig.5(c),horizontal line is the sequent number of frames, where a full line, dashed line and dashed dot line are for smokes, cars and passengers respectively.

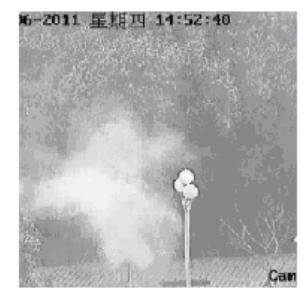

(a) An original image

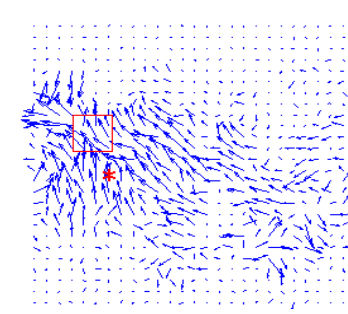

(b) optical flow field

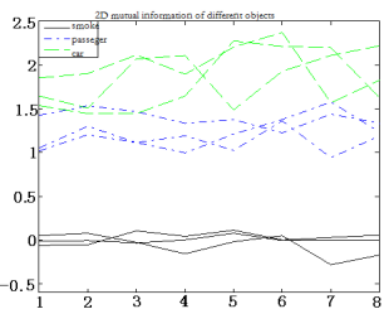

(c) the two dimensional mutual information

Fig.5 optical flow field and mutual information for motion

It is shown from Fig.5(c) that the mutual information of smoke regions takes much smaller values than that of cars and passengers as pixels' movements in smoke regions are of randomness while pixels' movements in cars or passengers have consistent directions and orientations. By using the descriptions of the two dimensional mutual information, the smoke objects are clearly separated from non-smoke objects. Finally, there is a test for contour variations shown in Fig.6.
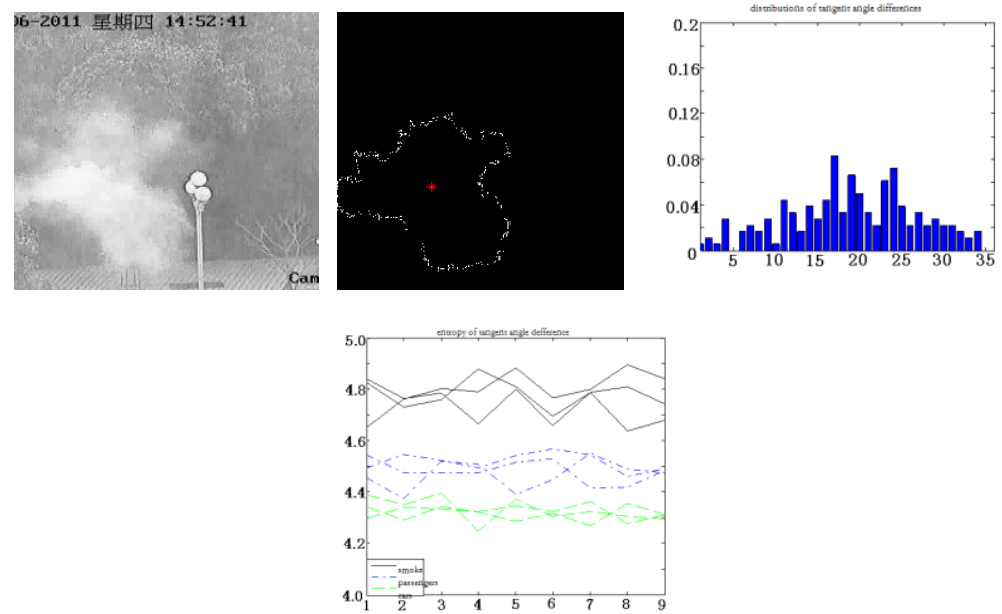

(a) Original image (b)the smoke contour (c)distribution of $\Delta Q_{k} \quad$ (d)entropy plots for many objects

Fig.6 experimental results for contour variations 
An original image and the extracted contour are shown in Fig.6 (a) and (b). The histogram of difference of tangent angles $\Delta \mathrm{Q}_{k}$ is given in Fig.6(c), shown that distribution of $\Delta \mathrm{Q}_{k}$ on the contour of a smoke object varies with time. It could be seen that three full lines in Fig.6 (d), corresponding to smoke objects, have higher entropy values. Meanwhile, dashed dot lines and dashed lines corresponding to passengers and cars take lower entropy values. From the Fig.6 (d), entropy of tangent angles $\left\{H\left(\Delta \mathrm{Q}_{\mathrm{k}}\right), k=1,2, \cdots, n\right\}$ seems to be a promising temporal-space feature to characterize smoke contour variations.

\section{Conclusion}

As for the descriptions of dynamic features, a novel method is composed of three temporal-space descriptors presented in the paper for smoke detections. The three descriptors are concatenated histograms of LBPs on the three orthogonal planes for dynamic textures, the two dimensional mutual information for motion chaos in a region, and entropy of difference of tangent angles on contours for dynamic contour variations. Experimental results are given to show the effectiveness of the proposed temporal-space features especially for the last two features. As for concatenated histograms of LBPs with dimension 256x3, they should be further processed on statistical measures to refine the feature descriptions.

\section{References}

1. Wang Z, Target-Tracking Based Early Fire Smoke Detection in Video. In:Proceedings of the IEEE

Workshop Image and Graphics.2009,1:172-176

2. S. Tom Au, Ma G, and Wang RS, An Optimal Temporal and Feature Space Allocation in Supervised Data Mining,In: proceedings of the international Multiconference of Engineers and Compter Scientists 2011 Vol.I, IMECS 2011, March 16-18,2011,Hong Kong

3. Amiaz T, Fazekas S, Chetverikov D and Kiryati N, Detecting regions of dynamic texture, In: proceeding of international conference on scale space and variational Methodism computer vision,2007, LNCS 4485,pp848-859

4. Ojala T, Multiresolution Gray-scale and Rotation Invariant Texture Classification with Local Binary Patterns._Proceedings of the IEEE Workshop Pattern Analysis and Machine Intelligence,2002,pp971-987.

5. Guo Y, Dynamic Texture Synthesis Using A Spatial Temporal Descriptor. Image Processing, 2009, pp $2277-2280$ 